A slight modification of the example given shows that if $N$ is chosen arbitrarily, there exists a limited region* having at least $N$ distinct points $O$ whose conjugates $D$ lie at infinity. $\dagger$

Theorem III becomes false if in the hypothesis the region $R$ is not assumed limited, for the reader may verify that no point $O$ of the region $R$ has its conjugate at infinity if $R$ is the entire plane slit along the positive half of the axis of reals from the point $z=0$ to infinity.

HARVARD UNIVERSITY

\title{
A NOTE ON LINEAR FUNCTIONALS
}

\section{R. P. BOAS, JR., $\ddagger$ AND J. W. TUKEY}

1. Introduction. The knowledge of the general form of linear functionals in a given abstract space $\S$ is of value in many problems. In some cases (notably in the theory of moment problems) the applications are not to the given space, but to its conjugate space; for example, since the general linear functional on $L$, the space of functions $x=x(t)$ integrable on $(0,1)$, has the form

$$
f(x)=\int_{0}^{1} x(t) a(t) d t, a \text { measurable and essentially bounded, } \|
$$

one can solve the moment problem

$$
\mu_{n}=\int_{0}^{1} t^{n} a(t) d t, \quad n=0,1,2, \cdots,
$$

for essentially bounded functions $a . \|$ From the point of view of the theory of moment problems, it seems quite fortuitous that there

\footnotetext{
* For the unlimited region $R:|y| \leqq b>0$ of the $(x, y)$-plane, every point $(x, 0)$ has as conjugate the point at infinity.

$\dagger$ The referee points out that for any region the set of points $O$ whose conjugates $D$ lie at infinity is identical with the set of critical points of the function $r(a)$.

$\ddagger$ National Research Fellow.

$\S$ We use the terminology of S. Banach, Théorie des Opérations Linéaires, Warsaw, 1932.

\| S. Banach, op. cit., p. 65. The function $a$ is said to be essentially bounded if there is a number $M$ such that $|a(t)| \leqq M$ for almost all $t$ on $(0,1)$; we denote by $\sup ^{0}|a(t)|$ the greatest lower bound of such numbers $M$.

I S. Banach, op. cit., p. 75.
} 
should exist a space, containing the functions $t^{n},(n=0,1,2, \cdots)$, on which (1.1) is the general linear functional; and if one attempts to discuss the moment problem (1.2) when $a$ is, for example, continuous or integrable, ${ }^{*}$ one meets the difficulty that

$$
f(x)=\int_{0}^{1} x(t) a(t) d t, a \text { continuous or integrable, }
$$

is not the general linear functional on any space for which the general linear functional has yet been determined.

One is thus led to seek the spaces on which a given expression (in particular, (1.3)) can represent the general linear functional. The purpose of this note is to show that (1.3) can represent the general linear functional on no "interesting" space; it follows, by a result of $\mathrm{J}$. W. Tukey, $\dagger$ that necessary and sufficient conditions for the solubility of the moment problem (1.2), with $a$ continuous or integrable, cannot take the form which F. Riesz, E. Helly, and S. Banach have given for other moment problems. $\ddagger$

It has been shown by I. Gelfand $\S$ that the spaces $C$ and $L$ (whose elements are continuous and integrable functions, respectively, with the classical metrics) are not the conjugate spaces of any Banach spaces. Gelfand's results clearly neither include ours nor are included in them. Our problem is of quite a different character. In Gelfand's problem, a particular Banach space is given, and one asks whether or not it is the conjugate of some Banach space. In the problem of which we consider certain special cases, we are given two vector spaces $J$ and $K$, without topology, and a functional $(j, k)$ defined on the Cartesian product of $J$ and $K$, distributive\| with respect to each argument separately. We ask whether or not a topology (satisfying certain conditions) can be introduced into some linear subset $H$ of $J$ (also satisfying certain conditions) in such a way that $H$ becomes a Banach space with the properties that every linear functional on $H$ (with this topology) has the form $f_{k}=f_{k}(h)=(h, k), h \in H, k \in K$, and every functional of this form is linear on $H$. If such a set $H$ exists, a topology is induced in $K$, possibly with identification of some of the elements of $K$, by considering $K$ as the conjugate space of $H$. The topologies in $H$ and $K$ obtained in this way may be entirely different

* In the sense of Lebesgue.

$\dagger$ To be published later.

$\ddagger$ S. Banach, op. cit., pp. 57, 74-75.

$\S$ I. Gelfand, Zur Theorie abstrakter Funktionen, Comptes Rendus (Doklady) de l'Académie des Sciences de l'URSS, vol. 17 (1937), pp. 243-245.

$\|$ An operation is called distributive if it is additive and homogeneous. 
from those which one would naturally expect in those spaces. We note that in the theory of moment problems the topology of the vector spaces involved is quite extraneous, so that our problem arises naturally in connection with that theory.

2. The theorems. We first make precise the notion of "general linear functional."

Definition. Let $R$ be a topological vector space of elements $x$. Let $P$ be a space of elements $p$. Let $f_{p}(x)$ be a functional with domain $R$, defined for each $p$ in $P$. We say, "a general linear functional in $R$ is $f_{p}(x)$," if

(i) $f_{p}(x)$ is a linear functional for every $p$ in $P$, and

(ii) every linear functional $g(x)$ with domain $R$ is identically equal to some $f_{p}(x)$.

We do not consider the question of whether or not this representation of the general linear functional in $R$ is redundant; that is, whether $f_{p}(x)=f_{q}(x)$ for every $x$ in $R$ is possible with $p \neq q$.

We consider first the functional (1.3) with integrable $a$.

THEOREM 1. Let $R$ be a normed vector space whose elements are realvalued functions $x=x(t),(0 \leqq t \leqq 1)$, (with the usual definitions of operations on elements). Let $R$ contain an infinite subset of the functions $t^{n}$, $(n=1,2, \cdots)$. Then

$$
f_{a}(x)=\int_{0}^{1} x(t) a(t) d t, \quad a \text { integrable, }
$$

is not a general linear functional on $R$.

We know no necessary and sufficient conditions for (2.1) to be a general linear functional on a space $R$. It is clear that (2.1) is a general linear functional on any finite-dimensional subspace of $B$ (the space whose elements are essentially bounded measurable functions on $(0,1)$, with the essential upper bound as norm); another result of the same character is the following theorem:

THeOREM 2. Let $E_{n},(n=1,2, \cdots)$, be mutually disjoint measurable subsets of $(0,1)$, of positive measure. Let $x_{n}(t)$ be the characteristic function of $E_{n},(n=1,2, \cdots)$. Let $R$ be the normed space whose elements are all finite linear combinations of the $x_{n}(t)$, with $\|x\|=\sup _{0 \leqq t \leqq 1}|x(t)|$. Then a general linear functional on $R$ is (2.1).

It is clear that the representation (2.1) is redundant (in the sense explained above). 
For the functional (1.3) with continuous $a$, our result is the following theorem :

TheOREM 3. Let $R$ be a vector space of type $F$, whose elements are real-valued functions $x=x(t),(0 \leqq t \leqq 1)$, (with the usual definitions of operations on elements). Let $R$ contain all of the functions $t^{n}$, $(n=0,1,2, \cdots)$, and let $R$ have the properties that

(a) $|x(t)|$ is in $R$ if $x(t)$ is in $R$,

(b) $\lim _{n \rightarrow \infty} x_{n}(t)=\Theta$ implies $\lim _{n \rightarrow \infty}\left|x_{n}(t)\right|=\Theta$, where $\Theta$ denotes the zero element of $R$.

Then

$$
f_{a}(x)=\int_{0}^{1} x(t) a(t) d t, \text { a continuous, }
$$

is not a general linear functional on $R$.

3. Proof of Theorem 1. Let $R$ satisfy the conditions of Theorem 1 . Suppose, if possible, that (2.1) is a general linear functional on $R$. Let $y=y(t) \equiv 1,(0 \leqq t \leqq 1)$. Then, for any $x$ in $R$,

$$
f_{y}(t)=\int_{0}^{1} x(t) y(t) d t=\int_{0}^{1} x(t) d t
$$

exists; that is, $x$ is integrable. Furthermore, $x$ is essentially bounded, since otherwise, by a theorem of Lebesgue, $\dagger \int_{0}^{1} x(t) a(t) d t$ could not exist for every integrable $a(t)$.

If $\lim _{n \rightarrow \infty}\left\|x_{n}\right\|=0$, then

$$
f_{a}\left(x_{n}\right)=\int_{0}^{1} x_{n}(t) a(t) d t \rightarrow 0, \quad n \rightarrow \infty,
$$

for every integrable $a$, since $f_{a}$ is a linear functional. Consequently, by another theorem of Lebesgue, $\ddagger$

$$
\lim _{n \rightarrow \infty} \sup _{0 \leqq}^{0}\left|x_{n}(t)\right|=0
$$

that is, $\lim _{n \rightarrow \infty}\left\|x_{n}\right\|_{B}=0$, where $\|x\|_{B}$ is the norm of $x$ in the space $B$ (mentioned above). It follows that any linear functional on $B$ is a linear functional on $R$.

Let $g=g(x)$ be the functional, constructed by T. H. Hildebrandt, $\S$

* S. Banach, op. cit., chap. 3. We do not use the full force of this hypothesis.

$\dagger \mathrm{E}$. W. Hobson, The Theory of Functions of a Real Variable and the Theory of Fourier's Series, vol. 1, 1927, p. 581.

$\ddagger$ E. W. Hobson, op. cit., vol. 2, 1926, p. 438.

$\S \mathrm{T}$. H. Hildebrandt, On bounded linear functional operations, Transactions of this Society, vol. 36 (1934), pp. 868-875; p. 875. 
such that $g$ is linear on $B$ (and consequently on $R$ ), and

$$
g[x(t)]=x(1)
$$

for every continuous $x$. If $g$ had the form (2.1), we should have, in particular,

$$
g\left(t^{n}\right)=\int_{0}^{1} t^{n} a(t) d t=1
$$

for the infinite sequence of values of $n$ for which $t^{n}$ is in $R$. But $t^{n} \rightarrow 0$, $(n \rightarrow \infty)$, boundedly for $0 \leqq t<1$, so that $\lim _{n \rightarrow \infty} \int_{0}^{1} t^{n} a(t) d t=0$, which contradicts (3.1). Consequently (2.1) is not a general linear functional on $R$.

4. Proof of Theorem 2. Let $R$ be the space defined in the hypothesis of Theorem 2. Let $f$ be an arbitrary linear functional on $R$, and let $c_{n}=f\left(x_{n}\right),(n=1,2, \cdots)$. Since $R$ is a normed vector space, there exists a constant $M$ such that for any real numbers $h_{i},(i=1,2, \cdots)$, and any integer $n$,

$$
\left|\sum_{i=1}^{n} h_{i} c_{i}\right| \leqq M\left\|\sum_{i=1}^{n} h_{i} x_{i}\right\|=M \max _{1 \leqq i \leqq n}\left|h_{i}\right| *^{*}
$$

Take $h_{i}=\operatorname{sgn} c_{i},(i=1,2, \cdots)$. Then (4.1) implies that

$$
\sum_{i=1}^{n}\left|c_{i}\right| \leqq M, \quad n=1,2, \cdots
$$

and consequently that $\sum_{i=1}^{\infty}\left|c_{i}\right| \leqq M$. Define $a=a(t)$ by

$$
a(t)=\left\{\begin{array}{cl}
c_{i} \cdot\left|E_{i}\right|^{-1}, & t \in E_{i}, \\
0, & t \oplus \sum_{i=1}^{\infty} E_{i},
\end{array}\right.
$$

where $|E|$ denotes the Lebesgue measure of $E$. Then $a$ is integrable, since

$$
\int_{0}^{1}|a(t)| d t=\sum_{i=1}^{\infty} \int_{E_{i}}\left|c_{i}\right| \cdot\left|E_{i}\right|^{-1} d t=\sum_{i=1}^{\infty}\left|c_{i}\right| \leqq M .
$$

We have

$$
\int_{0}^{1} x_{n}(t) a(t) d t=c_{n}=f\left(x_{n}\right), \quad n=1,2, \cdots .
$$

* S. Banach, op. cit., p. 57. 
Since the $E_{n}$ are mutually disjoint and $f(x)$ is linear, (4.2) implies that

$$
f(x)=\int_{0}^{1} x(t) a(t) d t, \quad x \in R .
$$

5. Proof of Theorem 3. Let $R$ have the properties specified in Theorem 3, and suppose, if possible, that a general linear functional in $R$ has the form (2.2). Then, as in $\S 3$, any $x$ in $R$ is integrable. Define a function $b=b(t)$ by

$$
b(t)= \begin{cases}1, & 0 \leqq t \leqq \frac{1}{2} \\ 0, & \frac{1}{2}<t \leqq 1\end{cases}
$$

Then

$$
g=g(x)=\int_{0}^{1} x(t) b(t) d t
$$

is a distributive functional on $R$ but is not linear. For, if $g$ were continuous, we should have, for all $x$ in $R$ and some continuous $b_{1}(t)$,

$$
\int_{0}^{1} x(t)\left[b(t)-b_{1}(t)\right] d t=0
$$

and in particular

$$
\int_{0}^{1} t^{n}\left[b(t)-b_{1}(t)\right] d t=0, \quad n=0,1,2, \cdots
$$

but it is well known that (5.1) implies that $b(t)=b_{1}(t)$ for almost all $t$.

Since $g$ is not linear, there must exist elements $x_{n}$ of $R$, $(n=1,2, \cdots)$, such that $\lim _{n \rightarrow \infty} x_{n}=\Theta$, while $g\left(x_{n}\right) \geqq 1$. Let $c(t)=1$, $(0 \leqq t \leqq 1)$. Since $c(t) \geqq b(t) \geqq 0,(0 \leqq t \leqq 1)$, we have, for $n=1,2, \cdots$,

$$
1 \leqq g\left(x_{n}\right)=\int_{0}^{1} x_{n}(t) b(t) d t \leqq \int_{0}^{1}\left|x_{n}(t)\right| b(t) d t \leqq \int_{0}^{1}\left|x_{n}(t)\right| c(t) d t .
$$

However, $h(x)=\int_{0}^{1} x(t) c(t) d t$ is a linear functional; hence, by properties (a) and (b),

$$
\lim _{n \rightarrow \infty} \int_{0}^{1}\left|x_{n}(t)\right| c(t) d t=0 .
$$

This contradiction establishes Theorem 3.

Princeton University 